\title{
Using Mobile Health Intervention to Improve Secondary Prevention of Coronary Heart Diseases in China: Mixed-Methods Feasibility Study
}

Shu Chen ${ }^{1}$, MSc; Enying Gong ${ }^{1}$, MSc; Dhruv S Kazi ${ }^{2,3,4}$, MSc, MD; Ann B Gates ${ }^{5}$, BPharm (Hons); Rong Bai ${ }^{6,7}$, MD; Hua Fu ${ }^{8}$, PhD; Weixia Peng ${ }^{8}$, PhD; Ginny De La Cruz ${ }^{9}$, MSc; Lei Chen ${ }^{10}$, MD; Xianxia Liu ${ }^{10}$, MD; Qingjie $\mathrm{Su}^{11}$, MD; Nicolas Girerd ${ }^{12,13}$, MPH, MD, PhD; Kamilu M Karaye ${ }^{14}$, FWACP, MBBS, PhD; Khalid F Alhabib ${ }^{15}$, MBBS, FESC, FACC, FRCPC; Lijing L Yan ${ }^{1,16}, \mathrm{MPH}, \mathrm{PhD}$; JD Schwalm ${ }^{17,18}$, MD

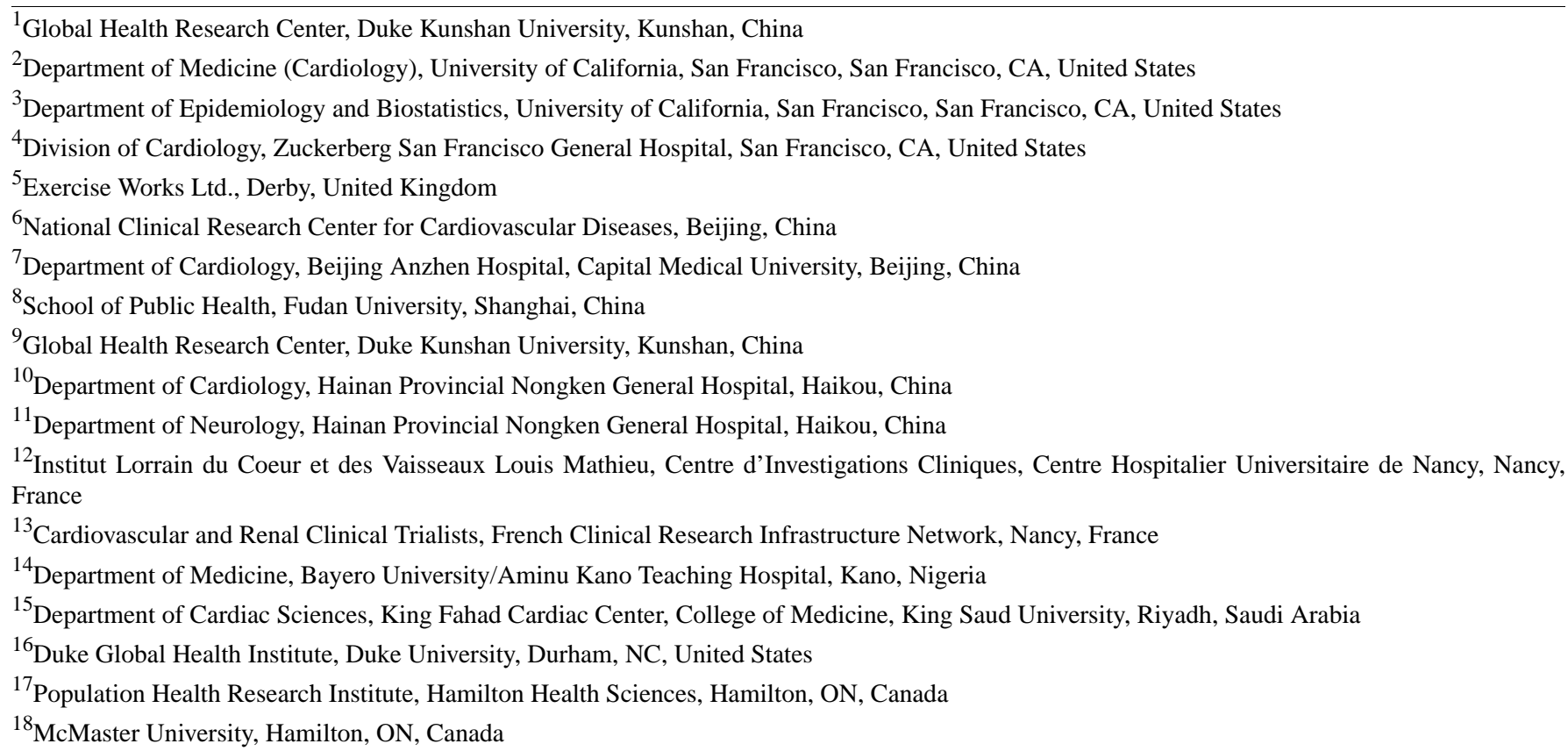

\section{Corresponding Author:}

Lijing L Yan, MPH, PhD

Global Health Research Center

Duke Kunshan University

No 8 Duke Avenue, Duke Kunshan University, Kunshan, Jiangsu, China

Kunshan, 215316

China

Phone: 8651236657057

Fax: 8651257728899

Email: lijing.yan@duke.edu

\section{Abstract}

Background: Coronary heart disease (CHD) is the leading cause of cardiovascular mortality worldwide, yet implementation of evidence-based strategies for secondary prevention remains suboptimal.

Objective: This study aimed to evaluate the feasibility, specifically the usability and acceptability, and estimate the preliminary effectiveness of a mobile health (mHealth) intervention targeting both physicians and patients to improve adherence to evidence-based medications and lifestyle modifications.

Methods: We conducted a 12-week pre-post interventional pilot study at two sites in Shanghai and Hainan, China. Physicians used the app designed in this study to prescribe evidence-based medicines and record patient information. Eligible and consenting 
patients received automatic text messages or voice calls 4 to 5 times per week for 12 weeks on medication adherence and healthy behaviors. Interviews were conducted among 10 physicians and 24 patients at the two sites for their thoughts on medication adherence and feedback on the usability and acceptability. Questions on usability and acceptability were also asked in a patient follow-up survey. With regard to estimating effectiveness, the primary outcome was medication adherence (as estimated by the Morisky Green Levine Scale) at 12 weeks. Secondary outcomes included physical activity, smoking status, fruits and vegetables consumption, and facility visit frequency.

Results: Interview findings and patient survey showed the good usability and acceptability of the intervention. Among 190 patients who completed the intervention, there was a significant increase in medication adherence (odds ratio [OR] 1.80, 95\% CI 1.14-2.85). The study also showed decrease of smokers' percentage $(-5 \%, P=.05)$, increase of daily vegetables consumption frequency ( $+0.3 /$ day, $P=.01$ ), and community health care center visit frequency ( +3 in 3 months, $P=.04)$. The following site-specific differences were noted: medication adherence appeared to increase in Hainan (OR 14.68, 95\% CI 5.20-41.45) but not in Shanghai (OR 0.61, 95\% CI 0.33-1.12).

Conclusions: Our study demonstrated that the intervention was feasible in both a tertiary care center and an urban community health center in China. Preliminary results from pre-post comparison suggest the possibility that provider and patient-linked mHealth interventions may improve medication adherence and lifestyle modifications among CHD patients, especially in resource-scarce settings. Randomized controlled trials are needed to verify the findings.

(JMIR Mhealth Uhealth 2018;6(1):e9) doi: 10.2196/mhealth.7849

\section{KEYWORDS}

coronary heart disease; secondary prevention; medication adherence; mobile applications; text messaging

\section{Introduction}

\section{Background}

Coronary heart disease (CHD) is the leading cause of cardiovascular mortality worldwide [1]. Despite a recent decline in high-income countries [2,3], CHD mortality continues to rise rapidly in low- and middle-income countries [4]. In China, CHD mortality was approximately 100 per 100,000 person in 2013 , ranking second only to stroke among causes of cardiovascular deaths $[5,6]$.

Clinical guidelines recommend a combined strategy of using evidence-based medicine and lifestyle modifications for secondary prevention. However, the implementation of these recommendations is suboptimal. The Prospective Urban Rural Epidemiology (PURE) study found that more than $50 \%$ of community-based patients from 17 high-, middle-, and low-income countries with known cardiovascular diseases did not take any medicines recommended by guidelines [7]. Even when patients have been initiated on evidence-based medicines at hospital discharge, the medication adherence is usually poor regardless of the socioeconomic status of the patients [8-10]. Evidence from a large multicenter retrospective analysis among more than 2901 CHD patients in China showed that less than $10 \%$ of patients used aspirin, clopidogrel, Angiotensin converting enzyme (ACE) inhibitors, or calcium antagonist at 1 year of hospital discharge [8]. Patients were also observed to have a high prevalence of residual lifestyle risk factors [9]. For example, $70 \%$ of men and $8 \%$ of women with CHD were active smokers in a large Chinese multicenter cross-sectional survey [9].

Addressing the implementation challenges of secondary prevention of CHD requires feasible, scalable, and cost-effective solutions. Recent advances in the widespread use of mobile phones and technology have made mobile health (mHealth) a promising solution. Short message service (SMS) for text messaging is one of the mHealth approaches that has been used in the management of diseases [10] such as asthma [11], human immunodeficiency syndrome (HIV) [12,13], malaria [14], diabetes [15], hypertension [10], and CHD [16,17]. However, the evidence of the effect of mHealth on medication adherence, diet, and physical activities remains inconclusive [10]. Furthermore, few studies have examined multifaceted interventions targeting both health care providers and patients for the improved delivery of secondary prevention of CHD [16].

\section{Information for the Adherence and Knowledge Exchange Heart Disease Medicines Study}

We designed and implemented the Adherence and Knowledge Exchange heart disease medicines study (TAKEmeds study) to examine whether an mHealth intervention can improve the patient adherence to evidence-based medications for the secondary prevention of CHD. The intervention includes a provider-facing mobile app guiding medicine prescription and a patient-directed text message or voice call system that promotes medication adherence and behavior modification to optimize secondary prevention of CHD. The specific purpose of this study was to pilot the intervention in China to examine its feasibility, including usability, acceptability, and preliminary effectiveness. This study follows the mHealth evidence reporting and assessment checklist [18].

\section{Methods}

\section{Study Design}

The TAKEmeds project was a pre-post multicenter interventional pilot study conducted in a community health care center in Shanghai and a tertiary hospital in Hainan Province, China (see Figure 1 for the flowchart of the study). The study was conceived and developed as a part of the World Heart Federation's Emerging Leader program. Implementation in China was led by the Global Health Research Center, Duke Kunshan University, in local partnership with the School of 
Public Health, Fudan University, and Hainan Provincial Nongken General Hospital (HPNGH). The study obtained ethical approval from the institutional review boards at Duke University Health System, Fudan University, and HPNGH. The trial was registered in the clinicaltrials.gov database on November 2015 (NCT02597205). The design process of the intervention tools has been previously reported [17].

\section{Study Sites}

The TAKEmeds study was conducted in a community health clinic in Longhua Street, Shanghai, and a tertiary care facility in Haikou, Hainan. Shanghai is a large metropolis with around 23 million people and the third highest gross domestic product (GDP) per capita in China, whereas Hainan is a tropical island province in southern China with around 8 million people, ranked 21 st out of 34 provinces in terms of GDP per capita [19]. The number of physicians per 1000 population in Shanghai was 2.17 versus 1.38 in Hainan in 2012 [20]. The Longhua Street Community Health Center in Shanghai serves 74,827 populations in 20,789 families. The Hainan Provincial Nongken General Hospital is one of the two tertiary hospitals serving Haikou, the capital city in Hainan, with around 1800 beds serving 590,300 residents. These two sites were selected because of their following distinctive features: primary health care center in a developed area and tertiary hospital in a developing area, which aids generalizability of our findings to disparate health systems across China.

\section{Study Participants}

Patients were eligible if (1) they had a history of a myocardial infarction (MI) or obstructive CHD (as clinically diagnosed by the treating physician), (2) they were physically and mentally able to manage their health care themselves, (3) they owned a mobile phone and were comfortable with receiving messages, (4) they were able and willing to provide informed consent, and (5) they were above 18 years of age. Patients who refused consent, participated in another study, or were severally ill with less than 3 months of expected survival were excluded.

In the community health center in Shanghai, patients were identified through the CHD patient list by general practitioners. In the tertiary hospital in Hainan, patients were identified through screening daily admissions and from cardiology outpatient clinic visits. The clinic was affiliated with the tertiary hospital. A promotion flyer that described the study aim and intervention and provided information about how to join the study was distributed to patients for recruitment. Patients were remunerated at the beginning and at end of study with a small gift (combined value 80 Ren Min Bi [RMB] [US \$13]).

Physicians were eligible if (1) they owned an Android mobile phone, (2) they took care of coronary artery diseases patients, and (3) they were willing to provide informed consent. Physicians that chose to participate in intervention were given a monetary compensation of around 200 RMB (around US \$32) in total.

Figure 1. The Adherence and Knowledge Exchange heart disease medicines (TAKEmeds) study flowchart.

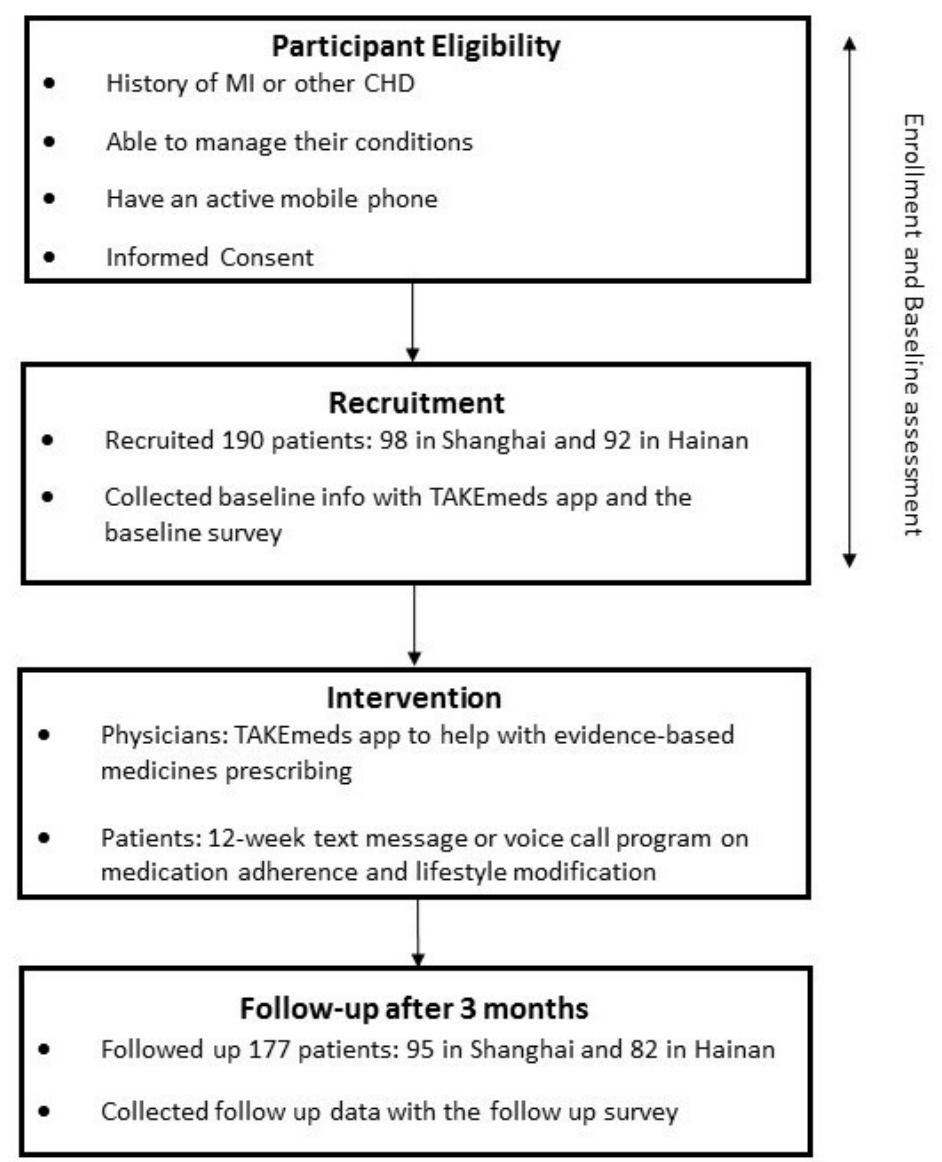




\section{Intervention}

The technology-enabled multifaceted intervention was developed to improve patient adherence to medications and modify their lifestyles. At the provider level, we developed an Android-based mobile app, named TAKEmeds, to support prescription of evidence-based medicines and facilitate patient recruitment. At the patient level, we developed a message bank with 60 messages that could be automatically sent to patients through a central server, nonrepetitively, over 12 weeks. These messages were based on current international guideline recommendations and evidence promoting medication adherence and lifestyle modifications. Patients who were active cigarette smokers at the time of enrollment also received tailored messages that help support smoking cessation. The TAKEmeds app and short messages were beta-tested and validated before the study rolled out. The app test version was validated by researchers and physicians, and the messages were tested among the employees in the development company. Details of app and messages development using principles of user-centered design have been described elsewhere [17].

After a brief training session, physicians used the app to record basic demographic and clinical information about eligible patients as well as to prescribe medications for secondary prevention of CHD. The TAKEmeds app made medication recommendations based on the guidelines for secondary prevention of MI published by the UK's National Institute for Health and Care Excellence (NICE) [21]. The NICE guideline was chosen as it was widely accepted and adopted internationally, and there are currently no national guidelines for MI secondary prevention in China. We also consulted a cardiologist in Beijing Anzhen Hospital (one of the coauthors) on its adaption in China before it was adopted. Physicians were able to choose medicines from the recommendations on the app after they entered time interval since heart disease onset $(\geq 0$ r $<1$ year). The app also provided information for physicians such as an overview of treatment options for MI patients with comorbidities and the importance of lifestyle changes. The flowchart of the app operational procedures and screenshots are presented in Figure 2, and more details can be found in the published development paper elsewhere [17].

At the time of enrollment, patients could choose to receive messages as short text messages or recorded phone calls. The phone calls were provided as an option for senior patients not comfortable with text messages, and they were machine recorded and shared the same contents (details of the messages and phone calls can be found in the paper published elsewhere) [17]. Messages covered the following 5 modules: medication adherence, physical activity, diet, smoking cessation, and general heart health. Example messages of the 5 modules are presented in Figure 3. We set the algorithm to send 4 to 5 messages per week to patients during weekdays for 12 weeks. Nonsmokers received one medication adherence message, one nutrition message, one exercise message, and one general heart health message on 4 random weekdays. Smokers received one additional message on smoking cessations. These messages were sent at one of the four random time slots: 9:00-9:10 AM, 12:00-12:10 PM, 3:00-3:10 PM, or 5:00-5:10 PM on the weekdays. As a part of the enrollment process, patients were also told the messages were one-way only (ie, patients could not respond to the messages with queries), and they could opt to stop receiving messages at any time by either informing their physicians or calling a given number.

\section{Outcome Measures}

The primary outcome was change in proportions of patients with different medication adherence status at 12 weeks compared with baseline. Medication adherence was measured by the 4-item Morisky Green Levine Scale [22]. Patients were classified into following 3 categories of adherence: low (score 3-4), intermediate (score 1-2), and high (score 0) [22].

Secondary outcomes included the following: (1) change in proportions of patients with different physical activity level, measured by the short-form version of the International Physical Activity Questionnaire [23]; (2) change in proportions of patients with different smoking status (classified as smoking every day, smoking occasionally, and never smoking); (3) change in the median of frequencies of patients consuming vegetables and fruits, calculated with the 6-item brief dietary assessment tool from the Behavioral Risk Factor Surveillance System fruit and vegetable dietary intake module [24]; and (4) change in the frequencies of patients visiting health facilities over the 3 months.

We conducted a patient survey at the end of the study to assess patient satisfaction toward the intervention, patient perceptions regarding the helpfulness of the intervention, and feedback regarding how they would prefer to receive messages in the future. To assess the affordability of the intervention, we collected basic costs of the messages or voice calls based on the charges of the carrier. We did not collect all costs incurred in this study, as it was not our intention to conduct a full economic analysis.

\section{Sample Size Estimates}

Sample size for this pilot study was primarily determined by budget and feasibility. The calculations were also done based on the primary outcome of medical adherence change at 12 weeks. Assuming the odds for patients with high medication adherence versus middle and low medication adherence (or high and middle medication adherence vs low medication adherence) were 10 times larger after the intervention, a total of 74 patients would have $80 \%$ power (two-sided alpha of .05). Allowing for $10 \%$ loss to follow-up and multiple sites, a total of 166 patients (83 patients in each site) would provide $80 \%$ power to detect the difference.

\section{Data Collection and Analysis}

\section{Quantitative Data}

We collected patient quantitative data from two sources. The first was the self-reported survey at baseline and 12 weeks at follow-up. The survey was administered by trained researchers in Shanghai and resident physicians in Hainan, and they collected data on primary and secondary outcome measures. In Shanghai, trained researchers visited the primary health care centers at baseline and follow-up to collect the data. The second was the TAKEmeds app into which demographic and clinical history were entered by physicians at the time of enrollment. 
Figure 2. Flowchart of the Adherence and Knowledge Exchange heart disease medicines (TAKEmeds) app operational procedures.
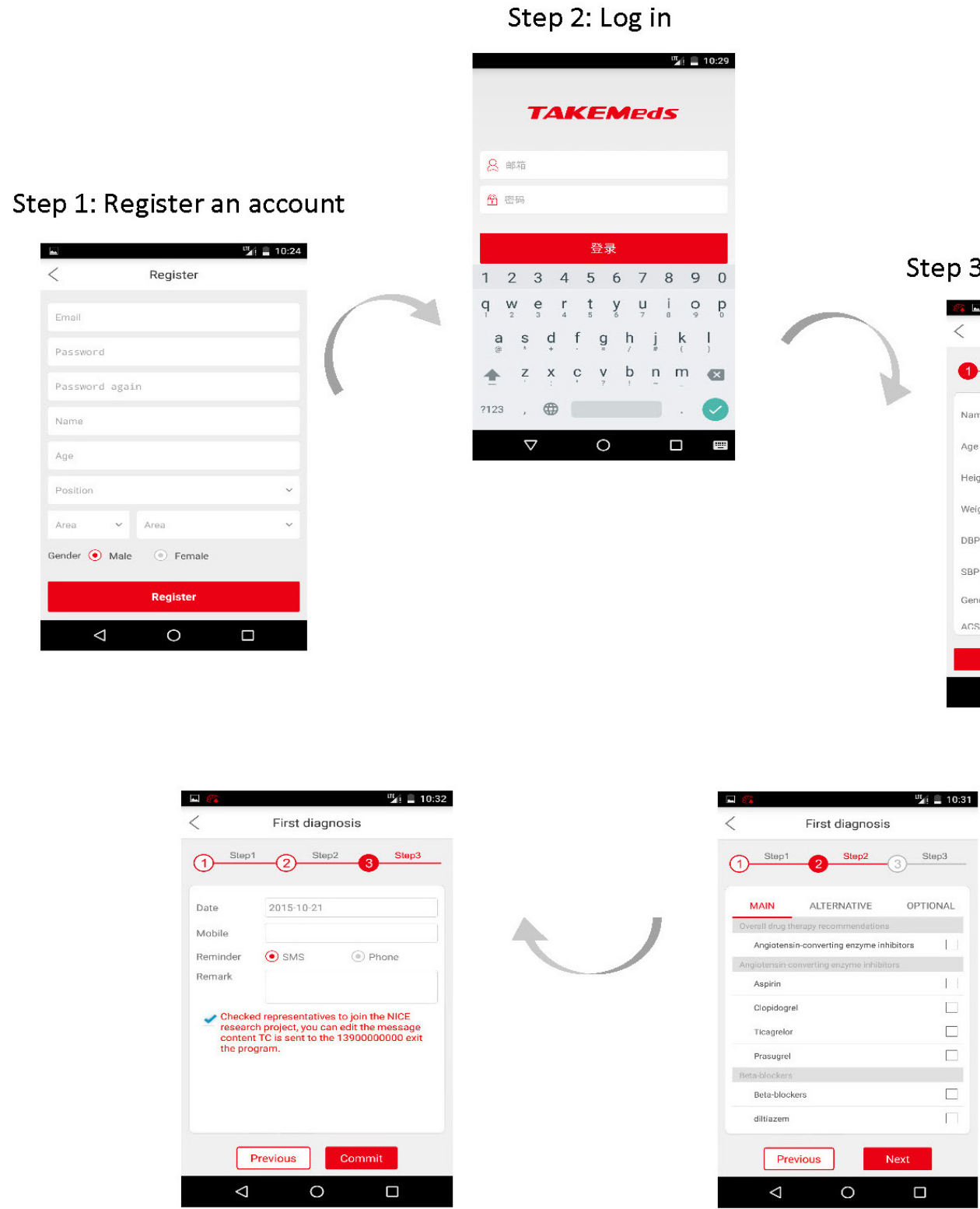

Step 5: Complete enrollment

Step 4: Prescribe 
Figure 3. Examples of text messages developed and used in the Adherence and Knowledge Exchange heart disease medicines (TAKEmeds) study.

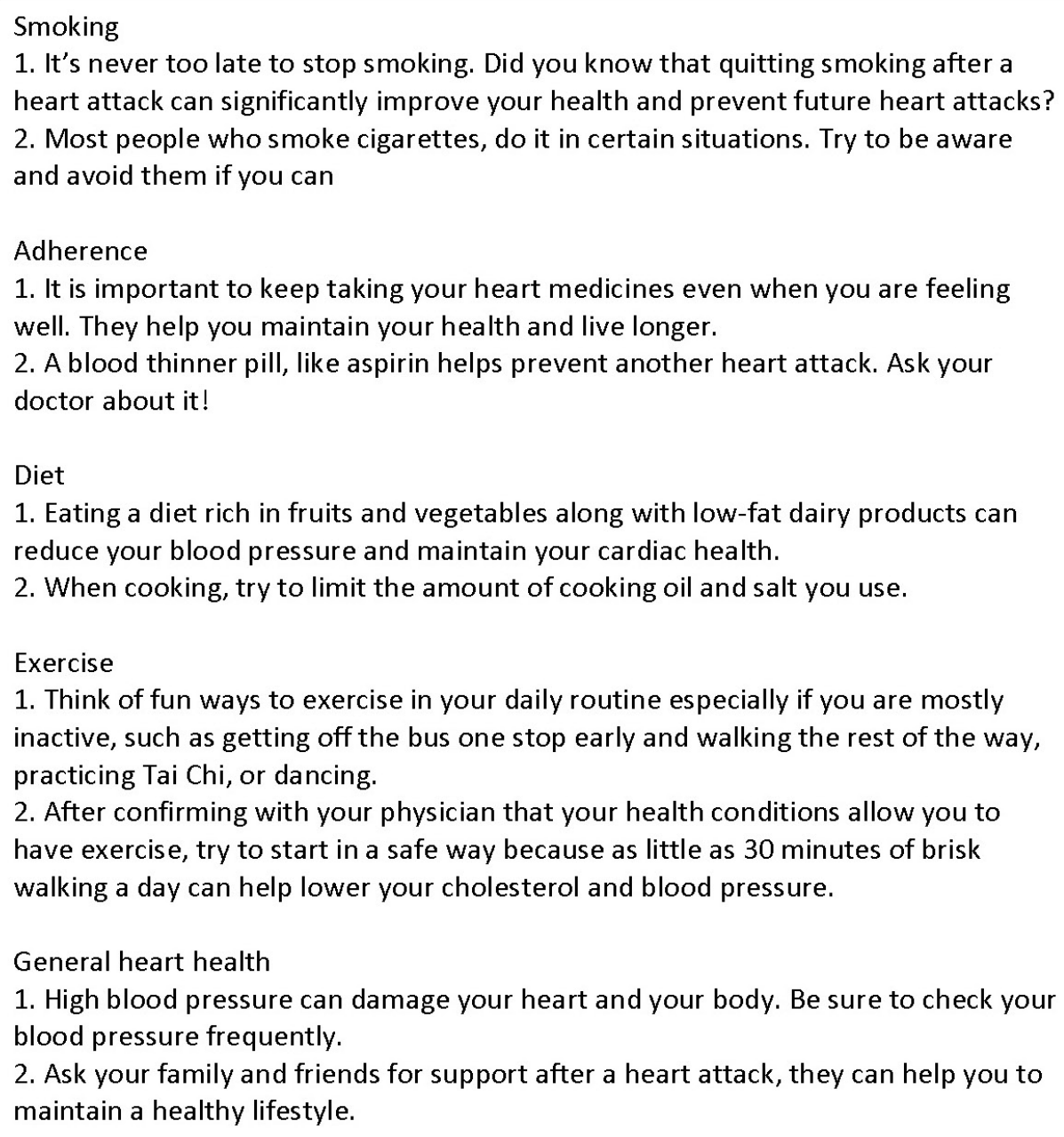

1. Think of fun ways to exercise in your daily routine especially if you are mostly inactive, such as getting off the bus one stop early and walking the rest of the way, practicing Tai Chi, or dancing.

2. After confirming with your physician that your health conditions allow you to have exercise, try to start in a safe way because as little as 30 minutes of brisk walking a day can help lower your cholesterol and blood pressure.

\section{General heart health}

1. High blood pressure can damage your heart and your body. Be sure to check your blood pressure frequently.

2. Ask your family and friends for support after a heart attack, they can help you to maintain a healthy lifestyle.

In addition, we estimated communication costs based on the recorded number of messages and the estimated duration of voice calls. The pre-post paired analyses were done at the individual patient level. As for the primary outcome, we built an ordinal (ordered) logistic regression model to test the intervention effect on medication adherence, and both univariate and covariate analyses were conducted. Time (before or after the intervention) was set as the dummy variable. Covariates in the model include age, gender, region, heart disease type, heart disease diagnosis time ( $\leq$ or $>1$ year), hypertension, diabetes status, and number of medications taken. Region was further treated as an interaction term to assess the outcome differences at the two sites. As for secondary outcomes, we used Wilcoxon test to compare the before and after change for physical activity, smoking status, fruits and vegetables consumption, and center visit frequency, and subgroup analysis was conducted. Analyses were conducted using STATA version 13.0 (StataCorp, College Station, TX, USA). All statistical tests were two-tailed with significance level set at .05.

\section{Qualitative Data}

The qualitative data were collected through process evaluations at the middle and end of the study. Two focus group discussions were conducted among physicians in Shanghai $(n=4)$ and Hainan $(\mathrm{n}=6)$, each lasting around 1 hour. Individual interviews were conducted among randomly selected patients $(n=24,12$ from each site) at both sites, each lasting $20 \mathrm{~min}$. The qualitative component was to understand the perceived barriers to improving medication adherence and their feedback on the usability and acceptability of the intervention.

Interviews and focus group discussions were recorded and transcribed. We used the thematic framework to analyze the qualitative results by using the NVivo version 10.0 qualitative data analysis software (QSR International Pty Ltd). Specifically, we coded the transcripts into different nodes of meaning and then grouped the nodes with similar meaning under one theme.

\section{Results}

Patient recruitment occurred from May 2015 to August 2015, and follow-up was completed by November 2015. A total of 190 patients were enrolled by 10 physicians, with 98 patients in Shanghai and 92 patients in Hainan. We successfully followed up 177 patients (follow-up rate 93.2\%) after the 12-week intervention. Loss to follow-up rate was $3.1 \%(n=3)$ in Shanghai and $10.9 \%(\mathrm{n}=10)$ in Hainan $(P=.03)$. No significant differences were observed between the profiles of the loss-to-follow patients 
compared with followed patients at the two sites combined or at each site. The results section presented findings of the preliminary effectiveness, usability, and acceptability of the intervention.

\section{Baseline Characteristics}

Baseline demographics, cardiac history, and medications of the 190 patients are presented in Table 1. Patients were $31.6 \%$ female, with a mean age of 67 years (SD 10). Patients in Hainan were 5 years younger than those in Shanghai (64 years vs 69 years, $P=.004$ ) with more severe CHD (a significantly higher proportion had a history of MI, and a higher proportion had been diagnosed with CHD within 1 year). All patients in Hainan took at least 2 cardiac medicines, whereas $79.6 \%$ of patients in Shanghai did so. However, fewer patients in Hainan had been previously diagnosed with hypertension $(47.8 \%$ vs $71.4 \%$ in
Shanghai). The vast majority of patients (94.2\%) chose to receive the information via text messages rather than via phone calls.

The mean age was 62 years (SD 10) for interviewed patients. On average, patients in Hainan (mean age: 57 years, SD 12) were younger than those in Shanghai (mean age: 68 years, SD 8 ). The percentage of male patients at both sites was $75.0 \%$. Six physicians in Hainan (all cardiologists) and 4 physicians in Shanghai (all general practitioners) participated in this study and focus group discussions. The mean age of physicians was 36 years (SD 5). Physicians in Shanghai (mean age 35 years, SD 4) were generally younger than those in Hainan (mean age: 37 years, SD 6); at both sites, $50.0 \%$ of the physicians were males. All Shanghai physicians were in practice for $\leq 10$ years, whereas Hainan physicians were in practice between 11 and 20 years.

Table 1. Demographic, health status, and medicine use of patients at baseline.

\begin{tabular}{|c|c|c|c|c|}
\hline Baseline characteristics & Total $(\mathrm{N}=190)$ & Shanghai $(\mathrm{N}=98)$ & Hainan $(\mathrm{N}=92)$ & $P$ value \\
\hline \multicolumn{5}{|l|}{ Gender, n (\%) } \\
\hline Female & $60(31.6)$ & $35(35.7)$ & $25(27.2)$ & \multirow[t]{2}{*}{.21} \\
\hline Male & $130(68.4)$ & $63(64.3)$ & $67(72.8)$ & \\
\hline Age in years, mean (SD) & $67(10)$ & $69(8)$ & $64(12)$ & .004 \\
\hline Diagnosed with hypertension, $\mathrm{n}(\%)$ & $114(60.0)$ & $70(71.4)$ & $44(47.8)$ & .001 \\
\hline Diagnosed with diabetes, $\mathrm{n}(\%)$ & $49(25.8)$ & $28(28.6)$ & $21(22.8)$ & .34 \\
\hline \multicolumn{5}{|l|}{ Heart disease type, $n(\%)$} \\
\hline $\mathrm{MI}^{\mathrm{a}}$ & $105(55.3)$ & $47(48.0)$ & $58(63.0)$ & \multirow[t]{2}{*}{.03} \\
\hline Non-MI & $85(44.7)$ & $51(52.0)$ & $34(37.0)$ & \\
\hline \multicolumn{5}{|l|}{ Time of diagnosis of heart disease, $n(\%)$} \\
\hline Within 1 year & $67(35.3)$ & $17(17.3)$ & $50(54.3)$ & \multirow[t]{2}{*}{$<.001$} \\
\hline More than 1 year & $123(64.7)$ & $81(82.7)$ & $42(45.7)$ & \\
\hline \multicolumn{5}{|l|}{ Number of cardiac medications, $n(\%)$} \\
\hline 1 & $20(10.5)$ & $20(20.4)$ & 0 & \multirow{3}{*}{$<.001$} \\
\hline 2 & $37(19.5)$ & $26(26.5)$ & $11(12.0)$ & \\
\hline 3 and more & $133(70.0)$ & $52(53.1)$ & $81(88.0)$ & \\
\hline \multicolumn{5}{|l|}{ Medication class, $\mathrm{n}(\%)$} \\
\hline $\mathrm{ACE}^{\mathrm{b}}$ inhibitors and $\mathrm{ARB}^{\mathrm{c}}$ & $43(22.6)$ & $27(27.6)$ & $16(17.4)$ & .09 \\
\hline Antiplatelets & $152(80.0)$ & $67(68.4)$ & $85(92.4)$ & $<.001$ \\
\hline Beta-blockers & $118(62.1)$ & $52(53.1)$ & $66(71.7)$ & .01 \\
\hline Aldosterone antagonists & $9(4.7)$ & 0 & $9(9.8)$ & .02 \\
\hline Statins and other lipid-lowering agents & $131(68.9)$ & $59(60.2)$ & $72(78.3)$ & .01 \\
\hline
\end{tabular}

${ }^{\mathrm{a}} \mathrm{MI}$ : myocardial infarction.

${ }^{\mathrm{b}} \mathrm{ACE}$ inhibitors: angiotensin-converting enzyme inhibitors.

${ }^{\mathrm{c}} \mathrm{ARB}$ : angiotensin receptor blockers. 
Table 2. Effect of the Adherence and Knowledge Exchange heart disease medicines (TAKEmeds) intervention on the primary outcome: medication adherence.

\begin{tabular}{|c|c|c|c|c|c|c|}
\hline Medication adherence & Baseline & Follow-up & $\begin{array}{l}\text { Unadjusted } \mathrm{OR}^{\mathrm{a}} \\
(95 \% \mathrm{CI})\end{array}$ & $P$ value & $\begin{array}{l}\text { Adjusted }^{\mathrm{b}} \text { OR } \\
(95 \% \mathrm{CI})\end{array}$ & $P$ value \\
\hline \multicolumn{7}{|l|}{ Combined, $n(\%)$} \\
\hline High & $107(61.5)$ & $121(69.5)$ & $1.74(1.09-2.78)$ & .02 & $1.80(1.14-2.85)$ & .01 \\
\hline Middle & $44(25.3)$ & $44(25.3)$ & & & & \\
\hline Low & $23(13.2)$ & $9(5.2)$ & & & & \\
\hline \multicolumn{7}{|l|}{ Shanghai, n (\%) } \\
\hline High & $61(64.9)$ & $47(50.0)$ & $0.71(0.42-1.22)$ & .22 & $0.61(0.33-1.12)$ & .11 \\
\hline Middle & $19(20.2)$ & $38(40.4)$ & & & & \\
\hline Low & $14(14.9)$ & $9(9.6)$ & & & & \\
\hline \multicolumn{7}{|l|}{ Hainan, n (\%) } \\
\hline High & $46(57.5)$ & $74(92.5)$ & $12.85(4.62-35.76)$ & $<.001$ & $14.68(5.20-41.45)$ & $<.001$ \\
\hline Middle & $25(31.3)$ & $6(7.5)$ & & & & \\
\hline Low & $9(11.3)$ & 0 & & & & \\
\hline
\end{tabular}

${ }^{\mathrm{a} O R}$ : Odds ratio.

${ }^{\mathrm{b}}$ Adjusted for age, gender, region, heart disease type, heart disease diagnosis time ( $<$ or $>1$ year), hypertension, diabetes status, and number of medications taken.

\section{Preliminary Estimate of Effectiveness: Primary Outcome}

\section{Quantitative Results}

The primary outcome results are presented in Table 2. We observed a significant improvement in medication adherence in participants postintervention, either before (odds ratio [OR] $1.74, P=.02$ ) or after adjusting for the covariates (OR 1.80, $P=.01)$. The percentage of participants reported with high medication adherence increased from $61.5 \%$ to $69.5 \%$ and with low medication adherence decreased from $13.2 \%$ to $5.2 \%$. Participants who took 2 (OR $3.11, P=.02$ ) or more than or equal to 3 medicines (OR 2.66, $P=.03$ ) tended to improve their medication adherence more significantly, compared with those who only took 1 medicine, after adjusting the covariates. Results of the full model can be found in Multimedia Appendix 1.

We identified a significant interaction between location of inclusion and intervention effect on medication adherence $(P<.001)$. Results of the model with region as the interaction term can be found in Multimedia Appendix 1. The unadjusted and adjusted outcomes were presented in Table 2 (descriptions here were adjusted outcomes only). In Hainan, there was a significant improvement in medication adherence (OR 14.68, $P<.001$ ), meaning that after the intervention, the odds for patients in Hainan with high medication adherence versus middle and low medication adherence (or high and middle medication adherence vs low medication adherence) were 14.68 times larger, given the other variables were held constant. This could be shown in the increase of percentage of participants with high medication adherence from $57.5 \%$ to $92.5 \%$. In contrast, we did not observe significant change in medication adherence in patients included in Shanghai (OR 0.61, $P=.11$ ).

\section{Qualitative Results}

The qualitative analysis of the patient and physician interviews revealed several factors that might lead to suboptimal medication adherence. Factors arising from the current flawed health system include the following: first, there existed no standard patient follow-up scheme, especially for cardiovascular patients. One physician stated the following:

There are follow-up interviews of hypertension and diabetes patients, while none for cardiovascular patients. [Physician, Shanghai, female, 33 years old]

Another physician stated the following:

It is our system's problem. There's no integrated NCD management scheme to follow-up with patients.

[Physician, Hainan, male, 35 years old]

Second, facilities suffered shortage of medicine supplies. One physician stated the following:

There are some other medicines. Although they are cheap, and we want to use them, there are no supplies. There's no on the market, and pharmaceutical companies are unwilling to produce

[Physician, Hainan, female, 38 years old]

Another physician stated the following:

It is difficult to prescribemedicines because community hospitals do not have some essential medicines for myocardial infarction patients. We lost them to tertiary hospitals. [Physician, Shanghai, female, 33 years old]

One physician stated the following:

For example, the community hospital only has short-acting Betaloc, instead of long-acting one. And 
other medicines are the same. It would be best if I did not have to go to large hospitals, but I have no other way. [Patient, Shanghai, female, 80 years old]

Third, the intense patient-physician relationship reduced patients' trust in physicians. One physician stated the following:

Patients would come only for prescriptions. They would think that community centers do not have good solutions. So it is hard to manage their conditions.

[Physician, Shanghai, male, 30 years old]

Another physician stated the following:

There's a crisis of trustiness. There are a lot of people think, we are aiming at something when we ask patient to take medicines. [Physician, Hainan, female, 38 years old]

Fourth, the unaffordability of the medicines kept patients from adhering to physicians. One physician stated the following:

I don't have much income as I have retired. It may be difficult, but there's no stipend for me. [Patient, Shanghai, male, 68 years old]

Another physician stated the following:

The patients may find the medicines are expensive and they cannot afford. So they have to quit it [Physician, Hainan, female, 38 years old]
Apart from factors attributing to current health system, another reason was that the prevention awareness among the patients was low. One physician stated the following:

Patients would only come and see a doctor when symptoms appear. [Physician, Shanghai, female, 33 years old]

In addition, medicines' side effects were another downside factor for patients to adhere.

\section{Preliminary Estimate of Effectiveness: Secondary Outcomes}

\section{Quantitative Results}

The secondary outcomes are presented in Table 3. We found an improving trend in patients' smoking status: the percentage of patients that reported no smoking increased from $83.0 \%$ to $87.5 \%(P=.05)$. We observed an increase in the daily frequency of consuming vegetables (from 2.4 to $2.7 /$ day, $P=.01$ ). The results showed an increase in community health care center visit frequency from 7 to 10 during the 12 weeks $(P=.04)$ in Shanghai. We did not find significant changes among patients in terms of fruits consumption $(P=.18)$ or physical activity $(P=.91)$.

\section{Qualitative Results}

The interviews and focus group discussions collected physicians' and patients' feedback on whether and how the intervention could help modify lifestyles and enhance physician-patient communication.

Table 3. Paired pre-post comparison of secondary outcomes.

\begin{tabular}{|c|c|c|c|c|c|c|c|c|c|}
\hline \multirow[t]{2}{*}{ Secondary outcomes } & \multicolumn{3}{|l|}{ Total } & \multicolumn{3}{|l|}{ Shanghai } & \multicolumn{3}{|l|}{ Hainan } \\
\hline & Baseline & Follow-up & $P$ value & Baseline & Follow-up & $P$ value & Baseline & Follow-up & $P$ value \\
\hline \multicolumn{10}{|l|}{ Physical activity, n (\%) } \\
\hline Highly active $^{\mathrm{a}}$ & $14(8.0)$ & $17(9.7)$ & .91 & $7(7.4)$ & $8(8.4)$ & .36 & $7(8.6)$ & $9(11.1)$ & .57 \\
\hline Minimally active ${ }^{\mathrm{b}}$ & $112(63.6)$ & $105(59.7)$ & & $69(72.6)$ & $62(65.3)$ & & $43(53.1)$ & $43(53.1)$ & \\
\hline Inactive $^{c}$ & $50(28.4)$ & $54(30.7)$ & & $19(20.0)$ & $25(26.3)$ & & $31(38.3)$ & $29(35.8)$ & \\
\hline \multicolumn{10}{|l|}{ Current smoking status, n (\%) } \\
\hline Everyday & $18(10.2)$ & $13(7.4)$ & .05 & $8(8.4)$ & $3(3.2)$ & .06 & $10(12.3)$ & $10(12.3)$ & .36 \\
\hline Seldom & $12(6.8)$ & $9(5.1)$ & & $6(6.3)$ & $8(8.4)$ & & $6(7.4)$ & $1(1.2)$ & \\
\hline Never & $146(83.0)$ & $154(87.5)$ & & $81(85.3)$ & $84(88.4)$ & & $65(80.2)$ & $70(86.4)$ & \\
\hline $\begin{array}{l}\text { Fruits consumption, median frequency/ } \\
\text { day }\end{array}$ & 0.5 & 0.6 & .18 & 0.6 & 0.8 & .65 & 0.3 & 0.6 & .17 \\
\hline $\begin{array}{l}\text { Vegetable consumption, median fre- } \\
\text { quency/day }\end{array}$ & 2.4 & 2.7 & .01 & 2.6 & 3.2 & .001 & 1.8 & 2.2 & .73 \\
\hline $\begin{array}{l}\text { Facility visit, median frequency } / 3 \\
\text { months }\end{array}$ & 3 & 3 & .22 & 7 & 10 & .04 & 1 & 2 & .69 \\
\hline
\end{tabular}

${ }^{\text {a } V i g o r o u s-i n t e n s i t y ~ a c t i v i t y ~ o n ~ a t ~ l e a s t ~} 3$ days, achieving a minimum of at least 1500 metabolic equivalent (MET)-minutes per week, OR 7 or more days of any combination of walking, moderate-intensity, or vigorous-intensity activities, achieving a minimum of at least 3000 MET-minutes per week. $\mathrm{b}_{3}$ or more days of vigorous activity for at least $20 \mathrm{~min}$ per day OR 5 or more days of moderate-intensity activity or walking for at least 30 min per day OR 5 or more days of any combination of walking, moderate-intensity, or vigorous-intensity activities, achieving a minimum of at least 600 MET-min per week.

${ }^{\mathrm{c}}$ Individuals who do not meet criteria for the above 2 categories are considered inactive. 
The comments we received were generally positive, albeit a few negative reviews remained. One patient stated the following:

After I began to receive message, I eat less meat when I was asked, and I work out when I am asked. [Patient, Shanghai, female, 67 years old]

Another patient stated the following:

It helps me receive more knowledge. For example, I received a message asked me to eat less meat. I don't like fish and I only eat meat. And the SMS asked me to eat less of it. [Patient, Shanghai, male, 74 years old]

One physician claimed that it was hard to change patients' lifestyle:

From my perspective, I would say that the effects are few. Because the lifestyle of patients is already mature, it is hard to be changed. And they don't pay much attention to it. [Physician, Shanghai, male, 33 years old]

Another physician stated that the intervention would take little effect in promoting communication with patients because they were too busy:

My patients are too many, and I don't have time to respond at all. [Physician, Hainan, male, 33 years old]

\section{Usability and Acceptability}

All interviewed patients in Shanghai and 10 out of the 12 interviewed patients in Hainan commented that the messages were easy to understand. All participated physicians commented that the app was easy to use; however, the use of prescription function was restricted. It took them 5 to 10 mins to set up a patient on the app.

Table 4. Perceived helpfulness of the intervention program and user feedback.

\begin{tabular}{|c|c|c|c|}
\hline Characteristics & Total, n (\%) & Shanghai, n (\%) & Hainan, $\mathrm{n}(\%)$ \\
\hline \multicolumn{4}{|l|}{ Helpful in } \\
\hline Medication adherence $(\mathrm{n}=152)$ & $123(80.9)$ & $68(74.7)$ & $55(90.2)$ \\
\hline Healthy diet $(n=173)$ & $132(76.3)$ & $69(75.8)$ & $63(76.8)$ \\
\hline Exercise $(\mathrm{n}=173)$ & $115(66.5)$ & $53(58.2)$ & $62(75.6)$ \\
\hline Smoking cessation $(\mathrm{n}=23)$ & $14(60.9)$ & $3(30.0)$ & $11(84.6)$ \\
\hline Communication with physicians ( $\mathrm{n}=173$ ) & $129(74.6)$ & $59(64.8)$ & $70(85.4)$ \\
\hline \multicolumn{4}{|l|}{ Preferred way of receiving messages $(n=171)$} \\
\hline Text messages & $111(64.9)$ & $84(92.3)$ & $27(33.8)$ \\
\hline Voice calls & $57(33.3)$ & $4(4.4)$ & $53(66.3)$ \\
\hline Other & $3(1.8)$ & $3(3.3)$ & 0 \\
\hline \multicolumn{4}{|l|}{ Preferred time of receiving messages $(n=173)$} \\
\hline 8:00-11:59 AM & $73(42.2)$ & $40(44.0)$ & $33(40.2)$ \\
\hline 12:00-12:59 PM & $3(1.7)$ & $2(2.2)$ & $1(1.2)$ \\
\hline 1:00-4:59 PM & $14(8.1)$ & $5(5.5)$ & $9(11.0)$ \\
\hline 5:00-9:00 PM & $24(13.9)$ & $9(9.9)$ & $15(18.3)$ \\
\hline Does not matter & $59(34.1)$ & $35(38.5)$ & $24(29.3)$ \\
\hline \multicolumn{4}{|c|}{ Preferred frequency of receiving messages $(n=171)$} \\
\hline$\geq$ Twice/day & $3(1.8)$ & $2(2.2)$ & $1(1.3)$ \\
\hline Once/day & $42(24.6)$ & $31(34.1)$ & $11(13.8)$ \\
\hline 5-6 times/week & $8(4.7)$ & $6(6.6)$ & $2(2.5)$ \\
\hline 3-4 times/week & $17(9.9)$ & $9(9.9)$ & $8(10.0)$ \\
\hline 1-2 times/week & $68(39.8)$ & $29(31.9)$ & $39(48.8)$ \\
\hline $1-3$ times/month & $33(19.3)$ & $14(15.4)$ & $19(23.8)$ \\
\hline \multicolumn{4}{|l|}{ Receiving messages in $(n=170)$} \\
\hline One-way only & $59(34.7)$ & $46(50.5)$ & $13(16.5)$ \\
\hline Two-way & $111(65.3)$ & $45(49.5)$ & $66(83.5)$ \\
\hline Satisfaction toward the program $(n=172)$ & $153(89.0)$ & $75(82.4)$ & $78(96.3)$ \\
\hline
\end{tabular}


The overall satisfaction rate with the program was $89.0 \%$ (see Table 4). Participants rated this intervention to be helpful with improving adherence to medications $(80.9 \%)$, dietary recommendations $(76.3 \%)$, increased physical activity $(66.5 \%)$, and smoking cessation $(60.9 \%)$. The majority of patients perceived that the app improved physician-patient communication $(74.6 \%)$.

We also received suggestions from physicians and patients on how to improve the intervention. Major suggestions included the following: incorporation of the intervention within the current health information system, further customizing message content to individual conditions, and talking slower on the recorded phone call.

A total of 11,534 messages were successfully delivered; however, because of local regulation policy, 166 messages failed to be sent at the beginning stage. All voice calls (720 in total) were successfully dialed and each voice call lasted for 1 to 2 min. During the course of the study, no patient requested cessation of message delivery.

The average cost charged by the carrier was approximately 0.10 RMB (around US \$0.015) per text message and 0.09 RMB (around US \$0.013) per voice call. To complete the 12-week intervention, it was estimated that for smokers, the average cost was 6 RMB (US \$0.9) for the text-message program and 5.4 RMB (US \$0.8) for the voice-call program; for nonsmokers, the costs were 4.8 RMB (around US \$0.74) and 4.3 RMB (around US \$0.66), respectively.

\section{Discussion}

\section{Summary of Findings}

To our knowledge, this is the first mixed-method study to target both providers and patients in China using mHealth technology to improve the secondary prevention of CHD. The intervention was feasible with easy-to-use products, low operational costs, and well accepted by patients, though the use of app prescription function was challenging for some physicians. We found that a multifaceted intervention that included a provider-facing Android app and a 12-week program of text messages or phone calls directed at patients with CHD was associated with an improvement in patient medication adherence (Hainan), smoking cessation (combined), and vegetable consumption (Shanghai) with notable regional differences that merit additional investigation.

\section{Use of Mobile Health Technology}

The ubiquity of mobile phones, especially in resource-limited settings, has made mHealth interventions an attractive option to modify health-related behaviors [25]. Quite a few studies have been conducted to improve the secondary prevention of CHD patients through text messages, and the evidence available seems consistent in supporting the positive effect of the intervention on medication adherence [26-28] and some have demonstrated the effectiveness on smoking cessation [29] and dietary habits [27,28].

In this study, we designed a mobile app for physicians and text messages for patients with the ultimate objective to improve medication adherence. Our working mechanism is to create a new follow-up platform through which patients could receive evidence-based support and recommendations from physicians to increase their compliance and improve their lifestyle.

\section{Interpretation of the Findings}

Interestingly, we found an association between the intervention and medication adherence among Hainan patients only. Possible drivers of the site-specific effect were differences in the underlying population, including burden of comorbidities, saturation of secondary prevention knowledge among patients, and hospitalization effect. The greater severity of heart disease conditions among patients recruited in Hainan (reflected in their MI proportion and medications number in Table 1) may account for the bigger changes in medication adherence, given that these patients likely had stronger motivations to follow treatment recommendations. We also observed from the patient interviews that the saturation of secondary knowledge among patients was lower, as they could only access secondary prevention knowledge through hospital physicians and this study. Four patients interviewed in Hainan claimed that this study was their only source for information regarding secondary prevention management. Shanghai patients, in comparison, could access the information through primary care providers, community health lectures, and family members. This would suggest that the benefit of messaging-based interventions might be greatest in low-resource settings with a dearth of reliable sources for health-related information. In addition, patients in Hainan experienced recent hospitalization, which might trigger them to better conform to physicians. Due to lack of control arm, we could not exclude the possibility that it was the recent hospitalization that provoked the change, which needs to be further verified in future studies. Though we found differences in patient age, hypertension history, heart diseases type, and time since diagnosis of heart disease between the two sites, the results suggest that those were not the driving factors of the difference in medication adherence.

The study results showed that the intervention might correlate with the improvement in smoking cessation, which is consistent with other mHealth trials [10,29-31]. We observed the association between intervention and the improvements in dietary habits, though it was unclear why the change of vegetables consumption was obvious in Shanghai only. The visit frequency to the community health center in Shanghai increased from 7 to 10 in 3 months. To regulate overprescribing behaviors of physicians in China, the essential medicines policy restricts that physicians in primary health care centers can only prescribe medicines at 1-month dosage. The increased frequency is a positive sign that the intervention promotes the physician-patient communication, and patients tended to have more frequent visit to the community health care centers.

\section{Barriers Encountered During Implementation}

Despite the solid mobile technology infrastructure in China, we encountered some technical barriers at implementation. Our text messages were initially suspended by the carrier for 3 to 5 days after pilot testing, as they were mistaken as fraud or commercial messages because of the high volume of messages. This was quickly rectified after we contacted the customer 
service departments of the telecommunications carrier and explained the study protocol. For some patients who chose to receive messages by phone call, the caller ID was not displayed correctly so the patients tended to incorrectly identify them as unsolicited advertising calls and hung up. This issue should be addressed in future studies by assigning a unique caller ID for incoming calls that would be shared with patients at enrollment.

The use of the app prescription function was partially restricted by the current Chinese health system. In the community health center, the function was limited as a result of the newly implemented essential medicine policy. The policy made a list of 307 essential medicines that could be stored at primary care centers and sold at low prices in 2009 and updated in 2012 to extend it to 520 medicines (each province can add medicines based on specific contexts) $[32,33]$. However, it turns out that it indirectly restricts the prescription rights of primary care providers as many truly essential medicines are not on the list. Therefore, some of the medicines suggested by the NICE guidelines, such as clopidogrel, could not be prescribed. Physicians in tertiary hospitals have full prescription rights; however, their heavy workload spared them little time to use the app.

\section{Strengths and Limitations of the Study}

Our study shows that the intervention is generally feasible. Our study targets both physicians and patients using technology-enabled intervention. In particular, the use of two disparate test sites - a community health center and a tertiary care hospital-greatly enhance the generalizability of our findings within the Chinese health care system. Differences in the findings from these two sites provided rich insights for future implementation research. Our understanding of the results was enhanced by the use of the mixed-method approach.

A principal limitation is that this study does not have a control group, which increases the risk that the change may not be attributed to the intervention only, although we built a regression model to control for possible confounders to mitigate the risk. Future studies with control arm are needed to evaluate its effectiveness in resource-limited settings. The small sample size and relatively short follow-up may preclude detection of small changes in adherence and lifestyle modifications and evaluation of persistence of efficacy over time. The self-reported nature of the outcomes means that results may be contaminated by recall or social likeability biases. Future studies may consider more objective measures, including pill counting for medication adherence, physiologic assessments (blood pressure, cholesterol, and glucose control), and clinical outcomes (MI, revascularization, and death). It may be necessary to include additional confounding variables, such as socioeconomic and psychosocial status, to sufficiently demonstrate the effects. Moreover, future studies should further examine fidelity to the intervention, that is, how many people actually read the messages or listened to the calls to accurately reflect their adherence to the intervention. Future studies could also record data related to number of eligible patients and reasons for nonparticipation to enhance the transparency of the recruitment process. Finally, the intervention was not incorporated within the current workflow of Chinese physicians, which was a barrier to its rapid and widespread adoption. Despite these limitations, this study provides experiences of implementing the TAKEmeds intervention model, its feasibility, and preliminary effectiveness results in two different settings in China.

\section{Conclusions}

Both international and national guidelines targeting medicine use and lifestyle modifications are well established to improve the secondary prevention of CHD; however, its uptake into clinical practice is far from optimal in China and around the world.

Such challenges require innovative, feasible, and cost-effective solutions. Harnessing the ubiquity of mobile phones and rapid advance in low-cost mobile technology, this study devised a multifaceted intervention package that targets both providers and physicians to increase the uptake of evidence-based secondary prevention of CHD in China. The results support the feasibility of the intervention: easy to use; well accepted by patients; and showing potential effect in improving medication adherence, smoking status, and possibly vegetables consumption as well as physician-patient interaction in community health centers, though we believe future studies with control group are needed to verify it before scaling up this intervention. We further found that the TAKEmeds intervention might have greater potential to improve the outcomes in resource-limited places such as Hainan, and further studies with control arms should verify the impact of the mHealth intervention across various economical settings. Although this study has several limitations, it provides a proof of concept for the role of mHealth in cardiovascular prevention. If these findings are confirmed in future studies with control arm and clinical endpoints, the intervention can be scaled up across the health system in China and other low-resource settings and can be adapted to other chronic diseases such as hypertension and diabetes.

\section{Acknowledgments}

The authors would like to acknowledge their academic partners including McMaster University and the Population Health Research Institute, Canada, which were involved in the study throughout the entire stage, and School of Public Health, Fudan University, which was in charge of the study implementation in Shanghai. The authors would also like to thank the Department of Cardiology, Hainan Nongken General Hospital, Haikou, Hainan Province, for their support of its implementation in Hainan. Specifically, the authors would like to thank the physicians in the Longhua Street Community Health Center and the physicians and residents in Hainan Nongken General Hospital that participated in and contributed to this study. The authors would also like to thank the Zhengjiang Innovation Zhejiang University Kunshan Innovation Institute for their help with app and message development. In addition, the authors acknowledge the contribution of Mr Yuliang Wang from School of Public Health, Fudan University; Dr Ye Sun from Hainan Nongken General Hospital for their research assistance and coordination; Prof Keith Dear and Dr Chaoyun Li from Duke Kunshan University; Ms Zhe Xu from John Hopkins University and Ms Yechu Hua from Duke University for their 
statistical support; Ms Minhui Yang for her qualitative research analysis support; Mr Heng Jiang for his help with references; and Louisa Hong and Kelly Sun from McMaster University for their support to develop the messages. Finally, the authors would like to thank World Heart Federation Emerging Leader Seed and the Duke Kunshan University Global Health Master Student Fieldwork for their funding support to this study. Travel support from the institution of each emerging leaders to attend the World Heart Federation (WHF) conference is also appreciated.

\section{Conflicts of Interest}

None declared.

\section{Multimedia Appendix 1}

Supplemental file (tables).

[PDF File (Adobe PDF File), 21KB-Multimedia Appendix 1]

\section{References}

1. Zhou M, Wang H, Zhu J, Chen W, Wang L, Liu S, et al. Cause-specific mortality for 240 causes in China during 1990-2013: a systematic subnational analysis for the Global Burden of Disease Study 2013. Lancet 2016 Jan 16;387(10015):251-272. [doi: 10.1016/S0140-6736(15)00551-6]

2. Writing Group Members, Mozaffarian D, Benjamin E, Go AS, Arnett DK, Blaha MJ, American Heart Association Statistics Committee, Stroke Statistics Subcommittee. Heart Disease and Stroke Statistics-2016 Update: A Report From the American Heart Association. Circulation 2016 Jan 26;133(4):e38-360. [doi: 10.1161/CIR.0000000000000350]

3. Nichols M, Townsend N, Scarborough P, Rayner M. Cardiovascular disease in Europe 2014: epidemiological update. Eur Heart J 2014 Nov 7;35(42):2950-2959. [doi: 10.1093/eurheartj/ehu299]

4. Reddy KS. Cardiovascular disease in non-Western countries. N Engl J Med 2004 Jun 10;350(24):2438-2440. [doi: 10.1056/NEJMp048024] [Medline: 15190135]

5. Zhang XH, Lu ZL, Liu L. Coronary heart disease in China. Heart 2008 Sep;94(9):1126-1131. [doi: 10.1136/hrt.2007.132423] [Medline: 18703693]

6. Jiang G, Wang D, Li W, Pan Y, Zheng W, Zhang HY, et al. Coronary heart disease mortality in China: age, gender, and urban-rural gaps during epidemiological transition. Rev Panam Salud Publica 2012 Apr;31(4):317-324. [Medline: 22652972]

7. Yusuf S, Islam S, Chow CK, Rangarajan S, Dagenais G, Diaz R, Prospective Urban Rural Epidemiology (PURE) Study Investigators. Use of secondary prevention drugs for cardiovascular disease in the community in high-income, middle-income, and low-income countries (the PURE Study): a prospective epidemiological survey. Lancet 2011 Oct 1;378(9798):1231-1243. [doi: 10.1016/S0140-6736(11)61215-4]

8. Bi Y, Gao R, Patel A, Su S, Gao W, Hu D, CPACS Investigators. Evidence-based medication use among Chinese patients with acute coronary syndromes at the time of hospital discharge and 1 year after hospitalization: results from the Clinical Pathways for Acute Coronary Syndromes in China (CPACS) study. Am Heart J 2009 Mar;157(3):509-516.e1. [doi: 10.1016/j.ahj.2008.09.026]

9. Liu H, Yu J, Pan C, Chen F, Li J, Hu D. [Cross-sectional study on the prevalence of metabolic syndrome in patients with coronary heart disease in China]. Zhonghua Yi Xue Za Zhi 2006 Aug 15;86(30):2095-2098. [Medline: 17064610]

10. de Jongh T, Gurol-Urganci I, Vodopivec-Jamsek V, Car J, Atun R. Mobile phone messaging for facilitating self-management of long-term illnesses. Cochrane Database Syst Rev 2012 Dec 12;12. [doi: 10.1002/14651858.CD007459.pub2]

11. Strandbygaard U, Thomsen SF, Backer V. A daily SMS reminder increases adherence to asthma treatment: a three-month follow-up study. Respir Med 2010 Feb;104(2):166-171. [doi: 10.1016/j.rmed.2009.10.003]

12. Mbuagbaw L, Thabane L, Ongolo-Zogo P, Lester RT, Mills E, Volmink J, et al. The Cameroon mobile phone SMS (CAMPS) trial: a protocol for a randomized controlled trial of mobile phone text messaging versus usual care for improving adherence to highly active anti-retroviral therapy. Trials 2011 Jan 7;12:5. [doi: 10.1186/1745-6215-12-5]

13. Lester RT, Ritvo P, Mills EJ, Kariri A, Karanja S, Chung MH, et al. Effects of a mobile phone short message service on antiretroviral treatment adherence in Kenya (WelTel Kenya1): a randomised trial. Lancet 2010 Nov 27;376(9755):1838-1845. [doi: 10.1016/S0140-6736(10)61997-6]

14. Ollivier L, Romand O, Marimoutou C, Michel R, Pognant C, Todesco A, et al. Use of short message service (SMS) to improve malaria chemoprophylaxis compliance after returning from a malaria endemic area. Malar J 2009 Oct 23;8:236. [Medline: 10.1186/1475-2875-8-236]

15. Kim HS, Kim NC, Ahn SH. Impact of a nurse short message service intervention for patients with diabetes. J Nurs Care Qual 2006;21(3):266-271. [Medline: 16816608]

16. Gandhi S, Chen S, Hong L, Sun K, Gong E, Li C, et al. Effect of mobile health interventions on the secondary prevention of cardiovascular disease: systematic review and meta-analysis. Can J Cardiol 2017 Feb;33(2):219-231. [doi:

10.1016/j.cjca.2016.08.017] 
17. Chen S, Gong E, Kazi DS, Gates AB, Karaye KM, Girerd N, et al. Development of a mobile phone-based intervention to improve adherence to secondary prevention of coronary heart disease in China. J Med Eng Technol 2016;40(7-8):372-382. [doi: 10.1080/03091902.2016.1213904]

18. Agarwal S, LeFevre AE, Lee J, L'Engle K, Mehl G, Sinha C, WHO mHealth Technical Evidence Review Group. Guidelines for reporting of health interventions using mobile phones: mobile health (mHealth) evidence reporting and assessment (mERA) checklist. BMJ 2016 Mar 17;352:i1174. [doi: 10.1136/bmj.i1174]

19. China Statistical Year Book. China: National Bureau of Statistics of China; 2015.

20. China Health Statistical Yearbook. China: National Bureau of Statistics of China; 2013.

21. National Institute for Health and Care Excellence. telfordccg. 2013. Myocardial infarction: Secondary prevention pathway URL: http://www.telfordccg.nhs.uk/your-health/medicines-management/prescribing-guidelines/cardiovascular/ myocardial-infarction/2339-secondary-prevention-myocardial-infarction-nice-pathway/file [accessed 2017-12-22] [WebCite Cache ID 6vttCpqId]

22. Morisky DE, Green LW, Levine DM. Concurrent and predictive validity of a self-reported measure of medication adherence. Med care 1986 Jan;24(1):67-74. [Medline: 3945130]

23. Craig CL, Marshall AL, Sjöström M, Bauman AE, Booth ML, Ainsworth BE, et al. International physical activity questionnaire: 12-country reliability and validity. Med Sci Sports Exerc 2003 Aug;35(8):1381-1395. [Medline: 12900694]

24. CDC. 2015. Surveillance of Fruit and Vegetable Intake Using the Behavioral Risk Factor Surveillance System URL: https:/ /www.cdc.gov/brfss/pdf/fruits vegetables.pdf [accessed 2017-12-18] [WebCite Cache ID 6vnf9mtIH]

25. Fjeldsoe BS, Marshall AL, Miller YD. Behavior change interventions delivered by mobile telephone short-message service. Am J Prev Med 2009 Feb;36(2):165-173. [doi: 10.1016/j.amepre.2008.09.040]

26. Park LG, Howie-Esquivel J, Chung ML, Dracup K. A text messaging intervention to promote medication adherence for patients with coronary heart disease: a randomized controlled trial. Patient Educ Couns 2014 Feb;94(2):261-268. [doi: 10.1016/j.pec.2013.10.027]

27. Pfaeffli Dale L, Whittaker R, Jiang Y, Stewart R, Rolleston A, Maddison R. Text message and Internet support for coronary heart disease self-management: results from the Text4Heart randomized controlled trial. J Med Internet Res 2015 Oct 21;17(10):e237. [doi: 10.2196/jmir.4944]

28. Varnfield M, Karunanithi M, Lee CK, Honeyman E, Arnold D, Ding H, et al. Smartphone-based home care model improved use of cardiac rehabilitation in postmyocardial infarction patients: results from a randomised controlled trial. Heart 2014 Nov;100(22):1770-1779. [doi: 10.1136/heartjnl-2014-305783]

29. Chow CK, Redfern J, Hillis GS, Thakkar J, Santo K, Hackett ML, et al. Effect of lifestyle-focused text messaging on risk factor modification in patients with coronary heart disease: a randomized clinical trial. J Am Med Assoc 2015;314(12):1255-1263. [doi: 10.1001/jama.2015.10945]

30. Free C, Knight R, Robertson S, Whittaker R, Edwards P, Zhou W, et al. Smoking cessation support delivered via mobile phone text messaging (txt2stop): a single-blind, randomised trial. Lancet 2011 Jun 30;378(9785):49-55. [doi:

10.1016/S0140-6736(11)60701-0]

31. Whittaker R, Borland R, Bullen C, Lin RB, McRobbie H, Rodgers A. Mobile phone-based interventions for smoking cessation. Cochrane Database Syst Rev 2009 Oct 07(4):CD006611. [doi: 10.1002/14651858.CD006611.pub2] [Medline: 19821377]

32. Ministry of Health of the People's Republic of China. moh.gov. 2009. Catalog of National Essential Medicines (Provision of Use for Primary Health Care Institutions) URL: http://www.moh.gov.cn/mohzcfgs/pgz/200908/42506.shtml [accessed 2017-12-26] [WebCite Cache ID 6vzkklCfK]

33. People's Republic of China Ministry of Health Order. SDA. 2012. National Essential Medicines List URL: http://www. sda.gov.cn/WS01/CL0053/79110.html [accessed 2017-12-26] [WebCite Cache ID 6vzkWuPJe]

\section{Abbreviations}

ACE: angiotensin-converting enzyme inhibitors.

ARB: angiotensin receptor blockers.

CHD: coronary heart disease

GDP: gross domestic product

HPNGH: Hainan Provincial Nongken General Hospital

MET: Metabolic Equivalent

mHealth: mobile health

MI: myocardial infarction

NICE: National Institute for Health and Care Excellence

OR: odds ratio

RMB: Ren Min Bi (Chinese Currency)

SMS: short message service

TAKEmeds: the Adherence and Knowledge Exchange heart disease medicines study 
Edited by G Eysenbach; submitted 11.04.17; peer-reviewed by M Tian, L Park, A Salzwedel; comments to author 22.07.17; revised version received 20.09.17; accepted 30.10.17; published 25.01.18

Please cite as:

Chen S, Gong E, Kazi DS, Gates AB, Bai R, Fu H, Peng W, De La Cruz G, Chen L, Liu X, Su Q, Girerd N, Karaye KM, Alhabib KF, Yan LL, Schwalm JD

Using Mobile Health Intervention to Improve Secondary Prevention of Coronary Heart Diseases in China: Mixed-Methods Feasibility Study

JMIR Mhealth Uhealth 2018;6(1):e9

URL: http://mhealth.jmir.org/2018/1/e9/

doi: 10.2196/mhealth.7849

PMID: 29371178

(C) Shu Chen, Enying Gong, Dhruv S Kazi, Ann B Gates, Rong Bai, Hua Fu, Weixia Peng, Ginny De La Cruz, Lei Chen, Xianxia Liu, Qingjie Su, Nicolas Girerd, Kamilu M Karaye, Khalid F Alhabib, Lijing L. Yan, JD Schwalm. Originally published in JMIR Mhealth and Uhealth (http://mhealth.jmir.org), 25.01.2018. This is an open-access article distributed under the terms of the Creative Commons Attribution License (https://creativecommons.org/licenses/by/4.0/), which permits unrestricted use, distribution, and reproduction in any medium, provided the original work, first published in JMIR mhealth and uhealth, is properly cited. The complete bibliographic information, a link to the original publication on http://mhealth.jmir.org/, as well as this copyright and license information must be included. 